\title{
Adaptive Hybrid Video Watermarking using WBCT and NMF
}

\author{
K.Kishore Kumar \\ Assistant Professor \& HOD, \\ ECE, Faculty of Science \& \\ Technology, IFHE University, \\ Hyderabad, 501203, India
}

\author{
Movva Pavani \\ Associate Professor, ECE \\ Aurora's Technological \& \\ Research Institute \\ Hyderabad, 500039, India
}

\author{
V.Seshu Babu \\ Research Engineer, \\ UURMI Systems, \\ Hyderabad, India.
}

\begin{abstract}
Watermarking techniques are mainly used for protecting intellectual property right. This paper proposes a new hybrid nonblind video watermarking technique using wavelet contourlet transform and nonnegative matrix factorization Wavelet transform processed images are losing edge information. The Contourlet transform has good approximation properties for smooth 2D functions and finds a direct discrete space construction. But its performance is considered to be redundant. There evolved wavelet based contourlet transform (WBCT), as a nonredundant version of the contourlet transform. WBCT is used for watermarking video frames. The non negative matrix factorization (NMF) is used as dimension reduction technique in watermarking. NMF is applied to low pass and directional high pass sub bands which results from WBCT of each original video frame and gray scale watermark images. Embedding action is performed in low pass sub-band of WBCT processed video frame. The hybrid scheme improves the performance of watermarking scheme. The experimental results shows that the proposed video watermarking scheme provides better video processing operations such as cropping, rotation, histogram equalization ,compression, variety of noises, frame dropping, frame averaging and frame swapping and etc.
\end{abstract}

Keywords: Contourlet Transform, Wavelet based Contourlet Transform, Nonnegative Matrix Factorization

\section{INTRODUCTION}

The popularity of digital video based applications [1] is accompanied by the need for copyright protection to prevent illicit copying and distribution of digital video. Copyright protection inserts authentication data such as ownership information and logo in the digital media without affecting its perceptual quality. In recent years, digital watermarking is one of the best potential tools for multimedia authentication by embedding some information into the digital production. This embedding information can be later extracted from or detected in the multimedia to make an assertion about the data authenticity. Digital watermarks remain intact under transmission/transformation, allowing us to protect our ownership rights in digital form. A watermarking algorithm consists of watermark structure, an embedding algorithm and extraction or detection algorithm. In multimedia applications, embedded watermark should be invisible, robust and have a high capacity. Invisibility refers to degree of distortion introduced by the watermark and its affect on the viewers and listeners. Robustness is the resistance of an embedded watermark against intentional attack and normal signal processing operations such as noise, filtering, rotation, scaling, cropping and lossy compression etc. Capacity is the amount of data can be represented by embedded watermark. Many digital watermarking schemes have been proposed for still images and videos [2]. Most of them operate on uncompressed videos [3-4], while others embed watermarks directly into compressed videos $[3,6]$. The work on video specific watermarking can be further found in [7-10]. Video watermarking introduces a number of issues not present in image watermarking. Due to inherent redundancy between video frames, video signals are highly susceptible to attacks such as frame averaging, frame dropping, frame swapping and statistical analysis. Video watermarking approaches can be classified into two main categories based on the method of hiding watermark bits in the host video. The two categories are: Spatial domain watermarking where embedding and detection of watermark are performed by directly manipulating the pixel intensity values of the video frame. Transform domain [11-13] techniques, on the other hand, alter spatial pixel values of the host video according to a predetermined transform and are more robust than spatial domain techniques since they disperse the watermark in the spatial domain of the video frame making it difficult to remove the watermark through malicious attacks like cropping, scaling, rotations and geometrical attacks. The commonly used transform domain techniques are Discrete Fourier Transform (DFT), the Discrete Cosine Transform (DCT) and the Discrete Wavelet Transform (DWT) among all many recent reviews are revealing that, DWT is very efficient transform technique for image as well as watermarking algorithms. The wavelet transform is very efficient in preserving multi resolution information of images but fails to preserve the curve structures, so the edges and some other smooth curves will lose continuous shapes and attains peace-wise continuous shapes, resulting slight disturbances in images. Likewise if wavelet transform is used in watermarking, the multimedia data will lose some of its artifacts. In the way towards solution of this problem there evolved Curvelet transform, Ridgeret transform, Contourlet transform. The contourlet transform has been introduced by Do and Vetterli (2003), to allow for different number of directions at each scale/resolution to achieve a critical sampling. The Contourlet transform has good approximation properties for smooth $2 \mathrm{D}$ functions, finds a direct discrete-space construction, and is therefore computationally efficient. For this purpose, Contourlet seems to be an appropriate candidate for image watermarking purpose. The Contourlet transform is a new directional transform which is capable of capturing contours and fine details in images. Originally it was defined in the discrete domain, but the authors proved its convergence in the continuous domain also. It is realized as a double iterated filter bank. The Discrete Contourlet Transform is also called 
as Pyramidal Directional Filter Bank (PDFB).Like wavelets, contourlets have a seamless translation between the continuous and the discrete domain via multiresolution framework and iterated filter banks. Consider Fig.1. the wavelet transform of a 2-D piecewise function with a smooth discontinuity curve. Due to separable construction, 2-D wavelet basis functions have supports on dyadic squares. Consequently, wavelets are good at isolating discontinuity points as only wavelets whose supports overlap with the discontinuity curve generate significant coefficients.

\subsection{Introduction to WBCT}

Although the wavelet transform is proved powerful in many signal and image processing applications such as compression, noise removal, image edge enhancement, and feature extraction, wavelets are not optimal in capturing the two dimensional singularities found in images. In particular, natural images consist of edges that are smooth curves and which cannot be captured efficiently by the wavelet transform. Therefore, several new transforms have been proposed for image signals. The contourlet transform is one of the new geometrical image transforms, which can efficiently represent images containing contours and textures. This transform uses a structure similar to that of curvelets, that is, a stage of subband decomposition followed by a directional transform. In the contourlet transform, a Laplacian Pyramid is employed for the first stage, while Directional Filter Banks (DFB) is used in the angular decomposition stage. Due to the redundancy of the Laplacian pyramid, the contourlet transform has a redundancy factor of $4 / 3$ and hence, it may not be the optimum choice for image coding applications.

The proposed WBCT achieves both radial and angular decomposition to an arbitrary extent and obeys the anisotropy scaling law of (width = length2). Compared to the aforementioned DFB based nonredundant transforms, the WBCT can easily be realized by applying DFB on the wavelet coefficients of an image. In this work, to improve the performance of the contourlet coder, we used the nonredundant WBCT in conjunction with an SPIHT algorithm to construct an embedded image coder. Due to differences in parent child relationships between the WBCT coefficients and wavelet coefficients, we developed an elaborated repositioning algorithm for the WBCT coefficients in such a way that we could have similar spatial orientation trees (the zero-trees) as the ones used for scanning the wavelet coefficients

\subsection{WBCT Decomposition}

Similar to the contourlet transform, the WBCT consists of two filter bank stages. The first stage provides subband decomposition, which in the case of the WBCT is a wavelet transform, in contrast to the Laplacian pyramid used in contourlets. The second stage of the WBCT is a directional filter bank (DFB), which provides angular decomposition. The first stage is realized by separable filter banks, while we implement the second stage using non-separable filter banks. For the DFB stage, we employ the iterated tree-structured filter banks using fan filters. For the first two levels, it is sufficient to use a simple quincunx filter bank. For higher levels of the wavelet decomposition, we use another building block, which is resampling followed by the quincunx. (Fig.1) To decrease artifacts due to Gibbs-like phenomenon in the DFB stage, we move down sampling and resampling to the end of the synthesis part and to the beginning of the analysis part, using the Nobel identities. We could achieve the anisotropy scaling law; that is, width $=$ length 2 . However, the wavelet filters are not perfect in splitting the frequency space to the low pass and high pass components, that is, not all of the directions in the HL image are vertical and in the LH image are horizontal, we use fully decomposed DFB on each band.

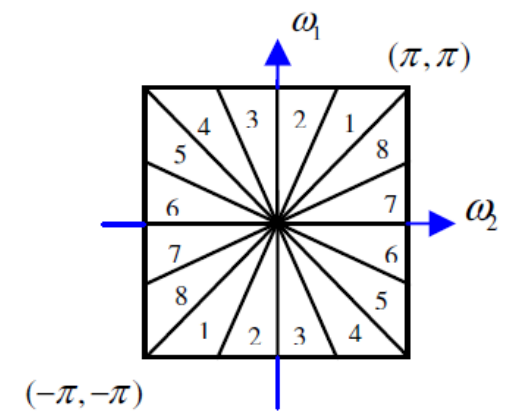

Fig. 1: Directional filter bank frequency partitioning

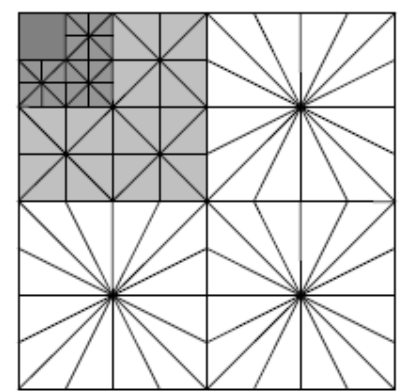

Fig. 2: A schematic plot of the WBCT using 3 dyadic wavelet levels and 8 directions at the finest level $(D N=8)$. The directional decomposition is overlaid the wavelet sub bands.

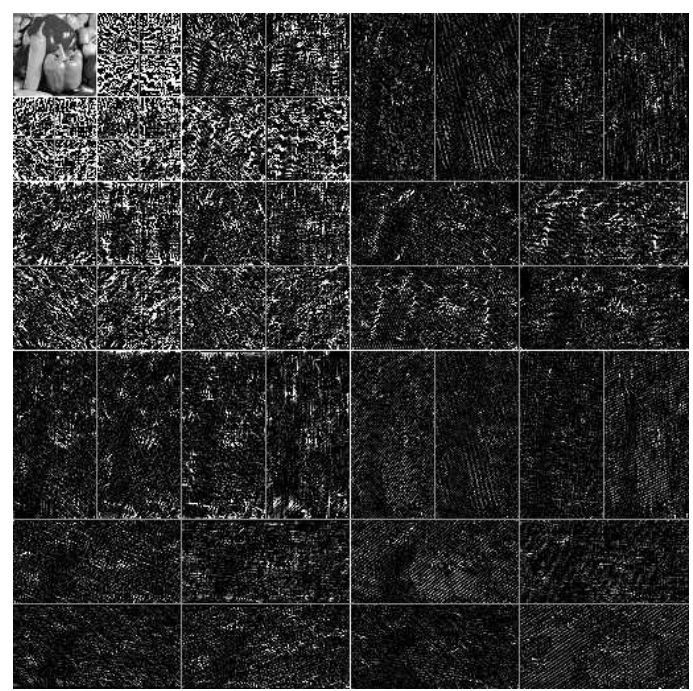

Fig. 3: The WBCT coefficients of the Peppers image. For better visualizing, the transform coefficients are clipped between 0 and 7.

\subsection{Nonnegative Matrix Factorization (NMF)}

One major drawback of SVD is that the basis vectors may have both positive and negative components and the data are represented as linear combinations of basis vectors of positive and negative coefficients. In many applications the negative components contradict physical realities and to address this problem, NMF approach was proposed to search for a representative basis with only negative vectors $[14,15]$.The $\mathrm{NMF}$ can be formulated as follows. Given a cover image $\mathrm{C}$ of size $\mathrm{m} \times \mathrm{m}$, we can approximately factorize $\mathrm{C}$ into the product of two non negative matrices $\mathrm{B}$ and $\mathrm{H}$ with sizes $\mathrm{m} \times \mathrm{r}$ and 
$\mathrm{r} \times \mathrm{m}$ respectively, that is $\mathrm{C}=\mathrm{BH}$, where $\mathrm{r} \leq \mathrm{m}$. The non negative matrix $\mathrm{B}$ contains the NMF basis vectors and the nonnegative weight matrix $\mathrm{H}$ contains the associated coefficients (nonnegative weights). To measure the quality of approximation factorization $\mathrm{C}=\mathrm{BH}$, a cost function between $\mathrm{C}$ and $\mathrm{BH}$ needs to be optimized subject to nonnegativity constraints on $\mathrm{B}$ and $\mathrm{H}$. This is done by minimizing the I-information divergence given by

$I(C \| B H)=\sum_{i j}\left(C_{i j} \log \frac{C_{i j}}{(B H)_{i j}}-C_{i j}+(B H)_{i j}\right)$

Which yields the following multiplicative update rules after updating one row of $\mathrm{H}$, we need o update the corresponding column of $\mathrm{B}$.

\section{PROPOSED METHOD}

There are many hybrid image watermarking schemes evolved using DWT+SVD,CT+SVD but SVD have a major weakness of SVD is that it produces low rank bases which do not respect the nonnegativity of the cover image. Nonnegative matrix factorization (NMF) was introduced in [14,15] to overcome this limitation without significantly increasing the error of the associated approximation and it has been shown to be an effective tool in many areas including spectroscopy [16].The embedding and extracting procedures as follows.

$H_{k j} \leftarrow H_{k j} \frac{\sum_{i} \frac{B_{i k} C_{i j}}{(B H)_{i j}}}{\sum_{i} B_{i k}} ; B_{i k} \leftarrow B_{i k} \frac{\sum_{j} \frac{H_{k j} C_{i j}}{(B H)_{i j}}}{\sum_{j} H_{k j}}$

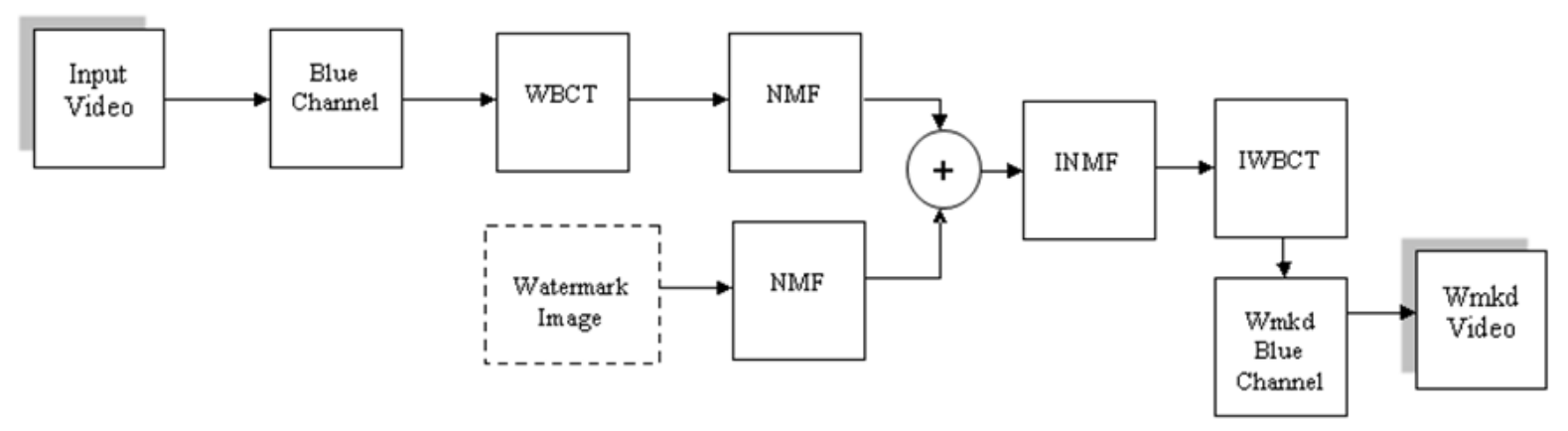

Fig. 4: Watermarking Procedure

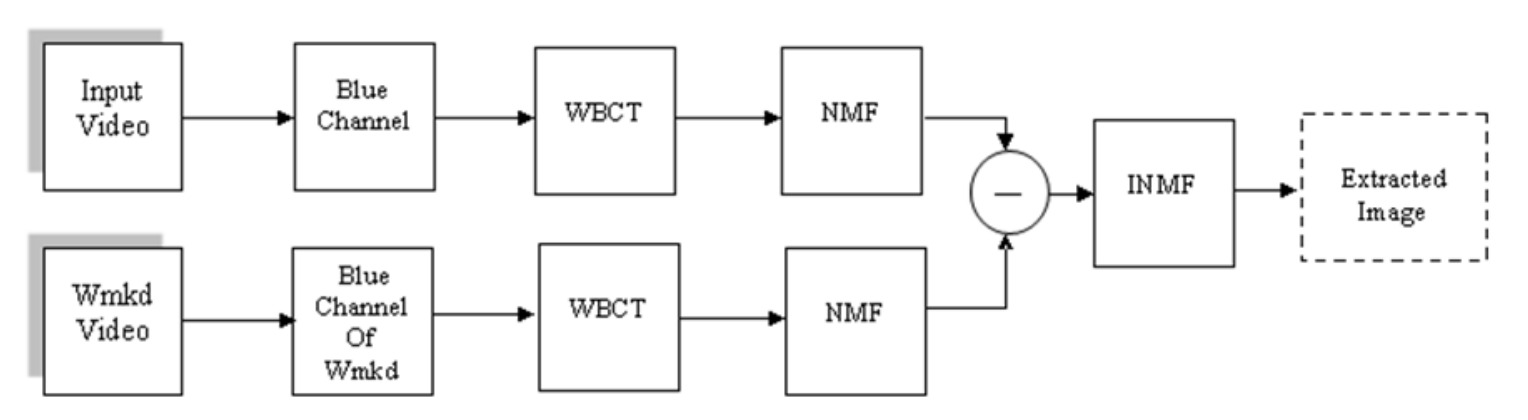

Fig. 5: Extracting Procedure

\subsection{Embedding Algorithm}

1. The input video is first extracted for its Blue frames and then the blue frames are send to wavelet based Contourlet transform.

2. Low frequency components are factorized into two $\mathrm{W}_{1}$ and $\mathrm{H}_{1}$ by Non-negative Matrix Factorization

3. The Gray scale image to be inserted is also factorized into $\mathrm{W}_{2}$ and $\mathrm{H}_{2}$ by NMF.

4. The $\mathrm{W}_{1}$ is normalized in between 0 and 1 and is termed as $\mathrm{M}_{1}$

$\mathrm{M}_{1}[\mathrm{i}]=\left(\mathrm{W}_{1}[\mathrm{i}]-\operatorname{maximum}\left(\mathrm{W}_{1}\right)\right) /\left(\operatorname{minimum}\left(\mathrm{W}_{1}\right)\right)$ $\left(\operatorname{maximum}\left(\mathrm{W}_{1}\right)\right)$

Where the matrices $\mathrm{B}$ and $\mathrm{H}$ are initialized as nonnegative random matrices and the updates are done alternatively that is
5. The weight matrix is obtained by Alpha $=0.05 * \mathrm{M}_{1}[\mathrm{i}]$

6. The embedding is performed as

$\mathrm{W}_{\text {new }}=\mathrm{W}_{1}+\mathrm{Alpha} \otimes \mathrm{W}_{2}$

$\otimes$--indicates element wise product.

7. After getting $\mathrm{W}_{\text {new }}$, using $\mathrm{H}_{1}$ the INMF results the watermarked coefficients of Blue Frame.

8. Inverse WBCT is applied to get back the watermarked Blue Frames and these will combined with Red and Green Frames of Original video to get back the Watermarked video.

9. After attaining this watermarked video, it is tested for imperceptibility by PSNR test. 


\subsection{Extracting Algorithm}

1. The input video and Watermarked videos are first extracted for its Blue frames and then the blue frames are send to wavelet based Contourlet transform.

2. Low frequency components are factorized into two $\mathrm{W}_{1}, \mathrm{H}_{1}$ and $\mathrm{W}_{\mathrm{wmkd}}, \mathrm{H}_{\mathrm{wmkd}}$ by Nonnegative Matrix Factorization.

3 The $\mathrm{W}_{\text {wmkd }}$ is normalized in between 0 and 1 and is termed as $\mathbf{M}_{\mathrm{wmkd}}$

$\mathrm{M}_{\mathrm{wmkd}}[\mathrm{i}]=\left(\mathrm{W}_{\mathrm{wmkd}}[\mathrm{i}]-\operatorname{maximum}\left(\mathrm{W}_{\mathrm{wmkd}}\right)\right) /$ $\left(\operatorname{minimum}\left(\mathrm{W}_{\mathrm{wmkd}}\right)\right)-\left(\operatorname{maximum}\left(\mathrm{W}_{\mathrm{wmkd}}\right)\right)$

4. The weight matrix is obtained by

5. The embedding is performed as

$$
\text { Alpha }=0.05 * \mathrm{M}_{\mathrm{wmkd}}[\mathrm{i}]
$$

$$
\mathrm{W}_{\text {new }}=\left(\mathrm{W}_{\mathrm{wmkd}^{-}}-\mathrm{W}_{1}\right) \varnothing \text { Alpha, }
$$

$\varnothing$--indicates element to element division

6. After getting $\mathrm{W}_{\text {new }}$, using $\mathrm{H}_{2}$ the INMF results the Extracted image

7. The Extracted image is processed for NCC test

8. To check the robust ness of the technique, the watermarked video is processed for various attacks and then extracting part is carried out, the resultant images are sent to NCC test.

\section{EXPERIMENTAL RESULTS}

In the experiments, we use the video "foremen.avi" of 294 frames of each size 256X256 as shown in the Table. 1 and four watermarks 'cameraman.jpg' of size 256 X 256 as watermark as shown in Table.1. The experiment is performed by taking scaling factor alpha as 0.05 to 0.5 in the steps of 0.01 .From the result one can decide there are no perceptibly visual degradations on the watermarked image shown in Table.1 with a PSNR of $75.29474 \mathrm{~dB}$. Extracted watermark without attack is shown in Table. 2 with NCC around unity. MATLAB 7.6 version is used for testing the robustness of the proposed method.

Various intentional and non-intentional attacks are tested for robustness of the proposed watermark algorithm includes JPEG,JPEG 2000 Compressions, Low Pass filtering, Rotation, Histogram Equalization, Median Filtering, Salt \&Pepper Noise, Weiner Filtering, Gamma Correction, Gaussian Noise Automatic Cropping, Dilation, Bit Plane Removal, Row Colum Copying, Row Colum sharpening etc

\begin{tabular}{|c|c|c|c|c|}
\hline & $\begin{array}{l}\text { Scene1(1 to } 30 \\
\text { frames) }\end{array}$ & $\begin{array}{l}\text { Scene2(31 to } 60 \\
\text { frames) }\end{array}$ & $\begin{array}{l}\text { Scene3(61 to } 90 \\
\text { frames) }\end{array}$ & $\begin{array}{l}\text { Scene1(91 to } 120 \\
\text { frames) }\end{array}$ \\
\hline \multicolumn{5}{|c|}{ Original frame } \\
\hline \multicolumn{5}{|c|}{ Watermarked frame } \\
\hline \multicolumn{5}{|l|}{$\begin{array}{l}\text { Watermark } \\
\text { image }\end{array}$} \\
\hline \multicolumn{5}{|c|}{ Extracted image } \\
\hline Avg NCC & 0.9999 & & & \\
\hline
\end{tabular}

Table.1 
Table.2

\begin{tabular}{|c|c|c|c|c|c|c|c|c|c|c|}
\hline Frame & \multicolumn{10}{|c|}{ PSNR } \\
\hline $1-10$ & 76.14057 & 75.69915 & 74.66502 & 74.72204 & 74.79629 & 74.75477 & 74.71477 & 74.77424 & 74.7503 & 74.56284 \\
\hline $11--20$ & 76.23202 & 75.79034 & 74.58144 & 74.66334 & 74.57799 & 74.5438 & 74.46827 & 74.57986 & 74.6361 & 74.58161 \\
\hline $21-30$ & 76.07615 & 75.65259 & 74.6691 & 74.51664 & 74.46548 & 74.52065 & 74.59307 & 74.42884 & 74.53111 & 74.55218 \\
\hline $31-40$ & 77.27716 & 76.91517 & 76.0377 & 76.02578 & 75.96215 & 76.01319 & 76.16936 & 76.07441 & 76.04684 & 76.16234 \\
\hline $41-50$ & 77.55965 & 77.23656 & 76.0824 & 76.08339 & 76.2302 & 76.26563 & 76.18805 & 76.14865 & 76.12649 & 76.08563 \\
\hline $51-60$ & 77.82675 & 77.34675 & 76.34675 & 76.34675 & 76.34675 & 76.34675 & 76.34675 & 76.34675 & 76.34675 & 76.34675 \\
\hline $61-70$ & 75.97838 & 75.50969 & 74.43788 & 74.47927 & 74.33767 & 74.52007 & 74.38869 & 74.50953 & 74.48353 & 74.3739 \\
\hline $71-80$ & 75.88682 & 75.39504 & 74.62832 & 74.34521 & 74.42062 & 74.30087 & 74.66329 & 74.39399 & 74.06279 & 73.92643 \\
\hline $81-90$ & 75.14503 & 74.72366 & 73.90226 & 73.88476 & 73.89646 & 73.8669 & 73.78158 & 73.83268 & 73.98014 & 73.70042 \\
\hline $91-100$ & 76.77032 & 76.17783 & 75.1769 & 75.23909 & 75.0916 & 75.22398 & 75.32262 & 75.08495 & 75.19634 & 75.20537 \\
\hline $101-110$ & 76.69094 & 76.25856 & 75.08504 & 75.09368 & 75.12894 & 75.01554 & 75.03784 & 75.037 & 75.1515 & 75.05393 \\
\hline $111-120$ & 76.66892 & 76.33599 & 75.36904 & 75.33767 & 75.2403 & 75.10932 & 74.99243 & 75.22094 & 75.2061 & 75.21121 \\
\hline & & & & & & 474 & & & & \\
\hline
\end{tabular}

\begin{tabular}{|c|c|c|c|c|c|}
\hline $\begin{array}{c}\text { Salt \& } \\
\text { Pepper Noise }\end{array}$ & $\begin{array}{c}\text { Scene1(1 to 30 } \\
\text { frames) }\end{array}$ & $\begin{array}{c}\text { Scene2(31 to } \\
60 \text { frames) }\end{array}$ & $\begin{array}{c}\text { Scene3(61 to } \\
90 \text { frames) }\end{array}$ & $\begin{array}{c}\text { Scene1(91 to } \\
120 \text { frames) }\end{array}$ & NCC \\
\hline Watermarked & & & &
\end{tabular}

\begin{tabular}{|c|c|c|c|c|c|}
\hline $\begin{array}{c}\text { Gaussian } \\
\text { Noise }\end{array}$ & $\begin{array}{c}\text { Scene1(1 to 30 } \\
\text { frames) }\end{array}$ & $\begin{array}{c}\text { Scene2(31 to } \\
60 \text { frames) }\end{array}$ & $\begin{array}{c}\text { Scene3(61 to } \\
90 \text { frames) }\end{array}$ & $\begin{array}{c}\text { Scene1(91 to } \\
120 \text { frames) }\end{array}$ & NCC \\
\hline Watermarked & & & &
\end{tabular}

Table.3

\begin{tabular}{|c|c|c|c|c|c|}
\hline Rotation & $\begin{array}{c}\text { Scene1(1 to 30 } \\
\text { frames) }\end{array}$ & $\begin{array}{c}\text { Scene2(31 to } \\
60 \text { frames) }\end{array}$ & $\begin{array}{c}\text { Scene3(61 to } \\
90 \text { frames) }\end{array}$ & $\begin{array}{c}\text { Scene1(91 to } \\
120 \text { frames) }\end{array}$ & NCC \\
\hline Watermarked & & &
\end{tabular}


International Journal of Computer Applications (0975 - 8887)

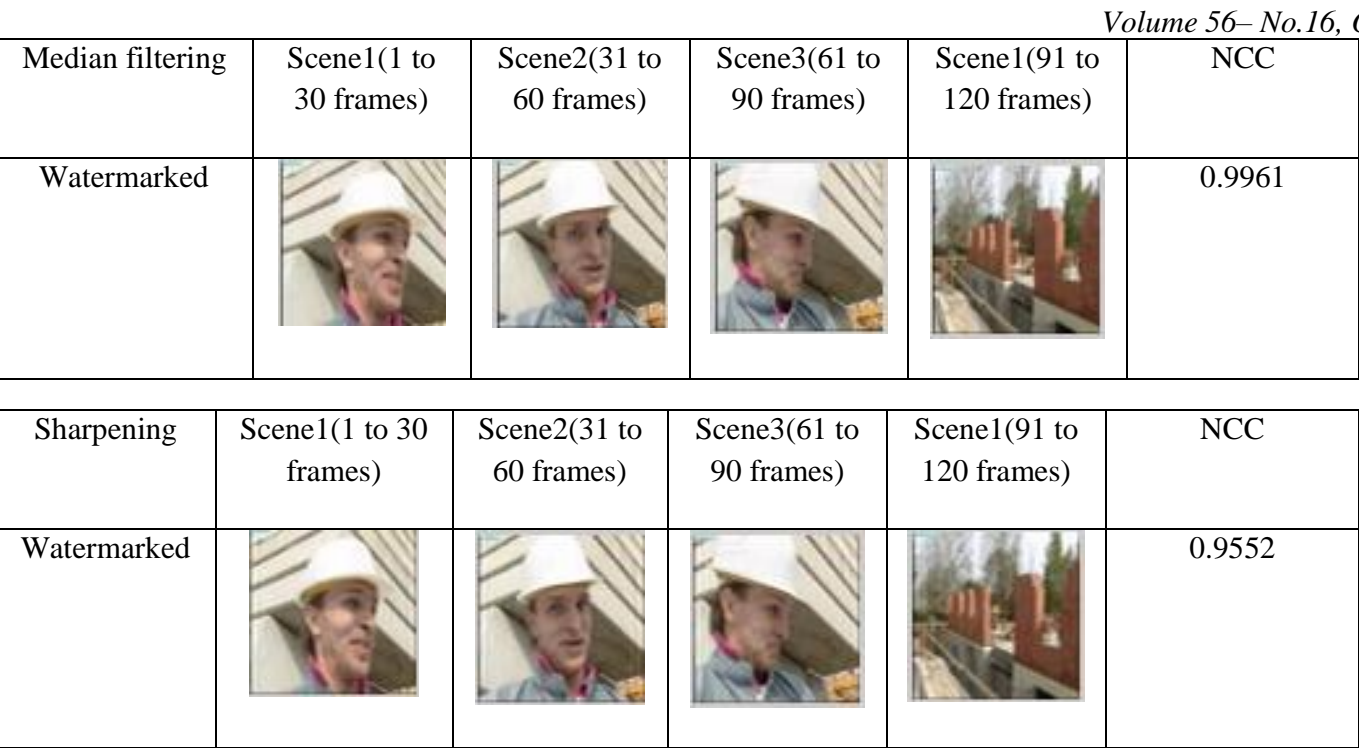

\begin{tabular}{|c|c|c|c|c|c|}
\hline $\begin{array}{c}\text { Contrast } \\
\text { Adjustment }\end{array}$ & $\begin{array}{c}\text { Scene1(1 to 30 } \\
\text { frames) }\end{array}$ & $\begin{array}{c}\text { Scene2(31 to } \\
60 \text { frames) }\end{array}$ & $\begin{array}{c}\text { Scene3(61 to } \\
90 \text { frames) }\end{array}$ & $\begin{array}{c}\text { Scene1(91 to } \\
120 \text { frames) }\end{array}$ & NCC \\
\hline Watermarked & & &
\end{tabular}

\begin{tabular}{|c|c|c|c|c|c|}
\hline Auto Crop & $\begin{array}{c}\text { Scene1(1 to 30 } \\
\text { frames) }\end{array}$ & $\begin{array}{c}\text { Scene2(31 to } \\
60 \text { frames) }\end{array}$ & $\begin{array}{c}\text { Scene3(61 to } \\
90 \text { frames) }\end{array}$ & $\begin{array}{c}\text { Scene1(91 to } \\
120 \text { frames) }\end{array}$ & NCC \\
\hline Watermarked & & &
\end{tabular}

Table.3

\begin{tabular}{|c|c|c|c|c|c|}
\hline $\begin{array}{c}\text { Weiner } \\
\text { Filtering }\end{array}$ & $\begin{array}{c}\text { Scene1(1 to 30 } \\
\text { frames) }\end{array}$ & $\begin{array}{c}\text { Scene2(31 to } \\
60 \text { frames) }\end{array}$ & $\begin{array}{c}\text { Scene3(61 to } \\
90 \text { frames) }\end{array}$ & $\begin{array}{c}\text { Scene1(91 to } \\
120 \text { frames) }\end{array}$ & NCC \\
\hline Watermarked & & &
\end{tabular}

\begin{tabular}{|c|c|c|c|c|c|}
\hline $\begin{array}{c}\text { Row Column } \\
\text { Removal }\end{array}$ & $\begin{array}{c}\text { Scene1(1 to 30 } \\
\text { frames) }\end{array}$ & $\begin{array}{c}\text { Scene2(31 to } \\
60 \text { frames) }\end{array}$ & $\begin{array}{c}\text { Scene3(61 to } \\
90 \text { frames) }\end{array}$ & $\begin{array}{c}\text { Scene1(91 to } \\
120 \text { frames) }\end{array}$ & NCC \\
\hline Watermarked & & &
\end{tabular}

\begin{tabular}{|c|c|c|c|c|c|}
\hline $\begin{array}{c}\text { Low Pass } \\
\text { Filtering }\end{array}$ & $\begin{array}{c}\text { Scene1(1 to 30 } \\
\text { frames) }\end{array}$ & $\begin{array}{c}\text { Scene2(31 to } \\
60 \text { frames) }\end{array}$ & $\begin{array}{c}\text { Scene3(61 to } \\
90 \text { frames) }\end{array}$ & $\begin{array}{c}\text { Scene1(91 to } \\
120 \text { frames) }\end{array}$ & NCC \\
\hline Watermarked & & &
\end{tabular}


Table.3

\begin{tabular}{|c|c|c|c|c|c|}
\hline $\begin{array}{c}\text { Frame } \\
\text { Dropping }\end{array}$ & $\begin{array}{c}\text { Scene1(1 to 30 } \\
\text { frames) }\end{array}$ & $\begin{array}{c}\text { Scene2(31 to } \\
60 \text { frames) }\end{array}$ & $\begin{array}{c}\text { Scene3(61 to } \\
90 \text { frames) }\end{array}$ & $\begin{array}{c}\text { Scene1(91 to } \\
120 \text { frames) }\end{array}$ & NCC \\
\hline Watermarked & & & & & \\
\hline
\end{tabular}

\begin{tabular}{|c|c|c|c|c|c|}
\hline Dilation & $\begin{array}{c}\text { Scene1(1 to 30 } \\
\text { frames) }\end{array}$ & $\begin{array}{c}\text { Scene2(31 to } \\
60 \text { frames) }\end{array}$ & $\begin{array}{c}\text { Scene3(61 to } \\
90 \text { frames) }\end{array}$ & $\begin{array}{c}\text { Scene1(91 to } \\
120 \text { frames) }\end{array}$ & NCC \\
\hline Watermarked & & & & \\
\hline
\end{tabular}

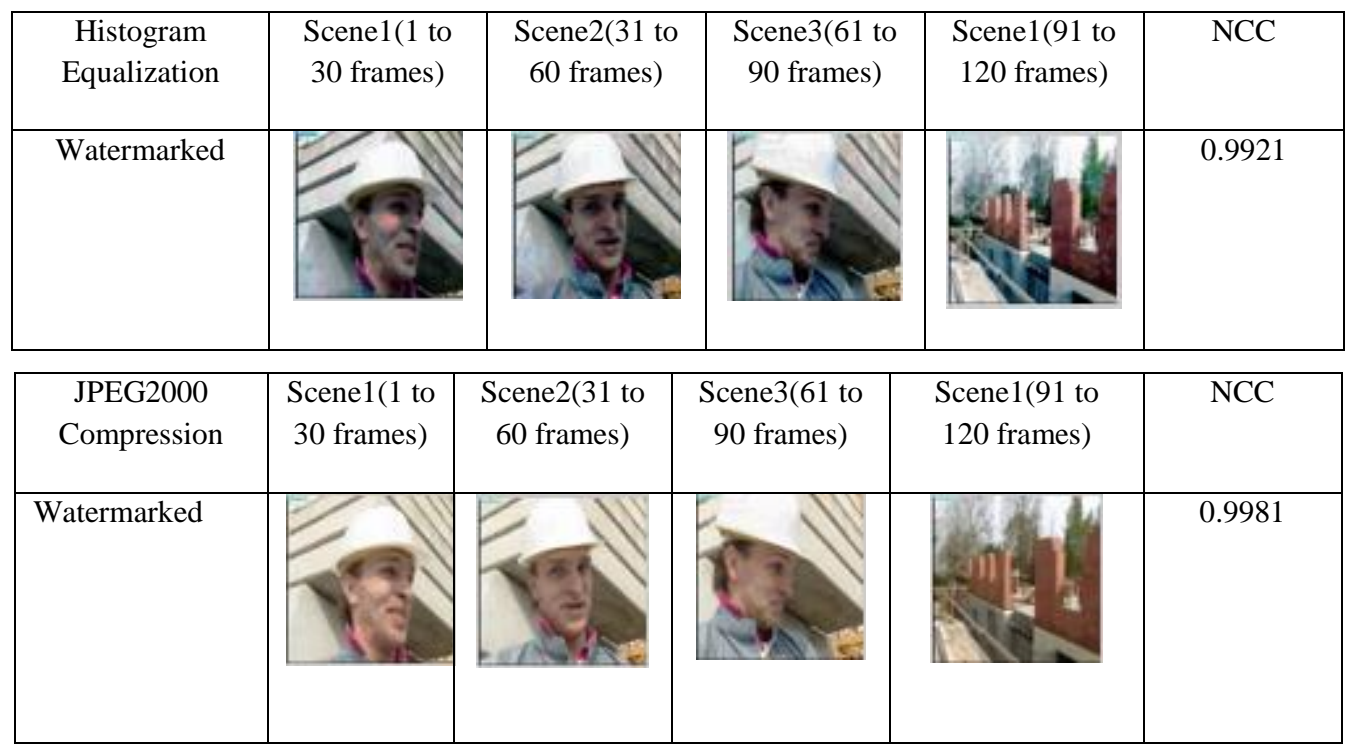

\begin{tabular}{|c|c|c|c|c|c|}
\hline $\begin{array}{c}\text { Gary scale } \\
\text { Conversion }\end{array}$ & $\begin{array}{c}\text { Scene1(1 to 30 } \\
\text { frames) }\end{array}$ & $\begin{array}{c}\text { Scene2(31 to } \\
60 \text { frames) }\end{array}$ & $\begin{array}{c}\text { Scene3(61 to } \\
90 \text { frames) }\end{array}$ & $\begin{array}{c}\text { Scene1(91 to } \\
120 \text { frames) }\end{array}$ & NCC \\
\hline Watermarked & & & & &
\end{tabular}

\begin{tabular}{|c|c|c|c|c|c|}
\hline Shearing & $\begin{array}{c}\text { Scene1(1 to 30 } \\
\text { frames) }\end{array}$ & $\begin{array}{c}\text { Scene2(31 to } \\
60 \text { frames) }\end{array}$ & $\begin{array}{c}\text { Scene3(61 to } \\
90 \text { frames) }\end{array}$ & $\begin{array}{c}\text { Scene1(91 to } \\
120 \text { frames) }\end{array}$ & NCC \\
\hline Watermarked & & &
\end{tabular}

\begin{tabular}{|c|c|c|c|c|c|}
\hline $\begin{array}{c}\text { Frame } \\
\text { Averaging }\end{array}$ & $\begin{array}{c}\text { Scene1(1 to 30 } \\
\text { frames) }\end{array}$ & $\begin{array}{c}\text { Scene2(31 to } \\
60 \text { frames) }\end{array}$ & $\begin{array}{c}\text { Scene3(61 to } \\
90 \text { frames) }\end{array}$ & $\begin{array}{c}\text { Scene1(91 to } \\
120 \text { frames) }\end{array}$ & NCC \\
\hline Watermarked & & & & &
\end{tabular}


The proposed algorithm is compared with Salwa A.K Mostafa algorithm [16], in which the watermarking is done by using wavelet transform + PCA. In our proposed method the PSNR obtained is 75.29474 and watermark image can survive up to attacks compared to Salwa A.K Mostafa algorithm.

In Table 3, the normalized correlation coefficient values for different attacks are shown with extracted watermark and attacked watermarked image. The quality and imperceptibility of watermarked video is measured by using PSNR. The PSNR is calculated separately for each scene using eq. 3 with respect to the respective color space of video. The final PSNR of watermarked image is taken as mean of PSNR obtained with all scenes. The similarity of extracted watermark with original watermark embedded is measured using NCC. The NCC is calculated using eq. (4) for the all scenes spaces and their mean is taken as the resultant Normalized Correlation coefficient.

\section{Peak Signal to Noise Ratio}

$P S N R=10 \log \left[\frac{\max (I(i, j))^{2}}{\sum_{N, M}\left(I^{\prime}(i, j)-I(i, j)\right)^{2}}\right] \ldots \ldots$ (3)

Normalized Correlation Coefficient

$$
N_{C C}=\frac{\sum_{m} \sum_{n}\left(A_{m n}-\bar{A}\right)\left(B_{m n}-\bar{B}\right)}{\sqrt{\left(\sum_{m} \sum_{n}\left(A_{m n}-\bar{A}\right)^{2}\right)\left(\sum_{m} \sum_{n}\left(B_{m n}-\bar{B}\right)^{2}\right)}}
$$

\section{CONCLUSIONS}

In this paper, a nonblind hybrid video watermarking scheme is proposed for video copyright protection using wavelet based contourlet transform and Nonnegative matrix factorization. The embedding is performed by adaptive changing the NMF factors of low frequency subband of blue frame of each video frame with respect to NMFs of watermark image. This technique has proven a great robustness with good NCC values on testing with various attacks.It is prudent with PSNR value that the imperceptibility is also very high with this technique. The proposed algorithm shows an excellent robustness to attacks like JPEG, JPEG2000 compressions, Gaussian Low pass filtering, Histogram equalization, Gray scale conversion, Salt and Pepper Noise, Shearing, Gamma correction, Cropping, Dilation, Weiner Filtering, Gaussian Noise, Rescaling, Rotation, Row Column Copying, Row Column Removal and Contrast adjustment. The proposed method shows higher robustness to maximum no of attacks compared to Salwa A.K Mostafa algorithm.

\section{REFERENCES}

[1] Yeo and M.M. Yeung, "Analysis and synthesis for new digital video applications,", International Conference on Image Processing (ICIP'97),vol. 1, pp.1,1997.

[2] G. Langelaar, I. Setyawan and R.Lagendijk, watermarking Digital Image and Video Data: A State of Art Overview," IEEE Signal Processing Magazine, vol.pp. 20-46, Sep.2000.
[3] F. Hartung and B. Girod, "Watermarking of uncompressed and compressed video," Signal Processing, 1998,vol. 66, no.3, pp.283-301.

[4] T. Khatib, A. Haj, L. Rajab, H. Mohammed, “ A Robust Video Watermarking Algorithm", Journal of Computer.science vol. 4,pp. 910-915, 2008

[5] T. Tokar, T. Kanocz, D. Levicky, "Digital watermarking of uncompressed video in spatial domain," 9th International Conference on Radioelectronica, IEEE, pp. 319-322, 2009

[6] J. Meng, and S.Chang, "Embedding Visible Video Watermarks in the Compressed Domain", International Conference on Image Processing, ICIP 98, Proceedings, vol.1,pp. 474-477,1998.

[7] P.W. Chan, M.R Lyu, R.T. Chin, "A Novel Scheme for Hybrid Digital Video Watermarking," IEEE Transactions on Circuits and Systems For Video Technology, vol. 15, no. 12, December 2005.

[8] G. Doërr, J.L. Dugelay , "A guide tour of video watermarking,"Signal Processing: Image Commun., vol. 18, no. 4, pp. 263-282,April 2003.

[9] Y. R. Lin, H.Y. Huang and W.H Hsu, "An embedded watermark technique in video for copyright protection", IEEE $18^{\text {th }}$ International Conference on Pattern Recognition, vol 00., pp. 795-798, 2006.

[10] C.V. Serdean, M.A. Ambroze., M. Tomlinson, J.G. Wade, "DWT Based Video Watermarking for Copyright Protection, Invariant to Geometrical Attacks", Proceedings of the 3rd International Symposium on Communication Systems Networks and Digital Signal Processing - CSNDSP'02, Stafford, UK, pp. 15-17 July 2002.

[11] I. J. Cox, J. Kilian, F. T. Leighton and T. Shamoon, "Secure spread spectrum watermarking for multimedia," IEEE Trans. on Image Processing, 1997, vol 6, no 12,pp. 1673-1687.

[12] C.H. Li and S.S. Wang, "Transform-Based Watermarking for Digital Images and Video," IEEE International Conference, June1999.

[13] S. Sinha, S. Pramanick, A. Jagatramka,P. Bardhan,D.K. Kole,A.Chakraborty, "Digital Video Watermarking using Singular Value Decomposition", Proceedings on IEEE EDS Student Paper Conference, pp. 53-56, 2011.

[14] D. Lee and H. Seung, "Learning the parts of objects by nonnegative matrix factorization," Nature,vol. 401, pp. 788-791, 1999

[15] D. Lee and H. Seung, "Algorithms for nonnegative matrix factorization," Adv. in Neural Info. Proc. Systems, 13, 2000.

[16]Salwa A.K.Mostafa, A.S.Tolba, F.M.Abdelkader, Hisham M.Elhindy, "Video Watermarking Scheme based on Principal Component Analysis and Wavelet Transform”, IJCSNS: International Journal of Computer Science and Network Security, Volume9, No: 8, August 2009. 